\title{
A Threat to a Virtual Hand Elicits Motor Cortex
}

\author{
Activation \\ Mar González-Franco ${ }^{1,5}$, Tabitha C. Peck ${ }^{1}$, \\ Antoni Rodríguez-Fornells ${ }^{2,3}$, Mel Slater 1,2, 4,5 \\ ${ }^{1}$ Event Lab, Faculty of Psychology, University of Barcelona, Spain \\ ${ }^{2}$ Institució Catalana de Recerca i Estudis Avançats (ICREA), Spain \\ ${ }^{3}$ Cognition and Brain Plasticity Group, Bellvitge Biomedical Research Institute \\ (IDIBELL) - Dept. Basic Psychology, University of Barcelona, Spain \\ ${ }^{4}$ Department of Computer Science, University College London, UK \\ ${ }^{5}$ IR3C Institute for Brain, Cognition and Behaviour, University of Barcelona, Spain
}

Running Title: Threat to a Virtual Hand

Corresponding author: Mel Slater, melslater@ub.edu 


\section{Abstract}

We report an experiment where participants observed an attack on their virtual body as experienced in an immersive virtual reality (IVR) system. Participants sat by a table with their right hand resting upon it. In IVR they saw a virtual table that was registered with the real one, and they had a virtual body that substituted their real body seen from a first person perspective. The virtual right hand was collocated with their real right hand. Event-related brain potentials (ERPs) were recorded in two conditions, one where the participant's virtual hand was attacked with a knife and a control condition where the knife only struck the virtual table. Significantly greater $\mathbf{P 4 5 0}$ potentials were obtained in the attack condition confirming our expectations that participants had a strong illusion of the virtual hand being their own, which was also strongly supported by questionnaire responses. Higher levels of subjective virtual hand ownership correlate with larger P450 amplitudes. Mu-rhythm Event Related Desynchronization (ERD) in the motor cortex, and Readiness Potential (C3-C4) negativity were clearly observed when the virtual hand was threatened -as would be expected if the real hand was threatened and the participant tried to avoid harm. Our results support the idea that eventrelated potentials (ERPs) may provide a promising non-subjective measure of virtual embodiment. They also support previous experiments on pain observation and are placed into context of similar experiments and studies of body-perception and body-ownership within cognitive neu roscience.

Key words: body ownership; rubber hand illusion; virtual reality; motor cortex; pain; ERPs 


\section{Introduction}

When someone anticipates that a knife might stab their hand that is resting on a table they would be likely to attempt to move the threatened hand out of the way. They would expect to feel considerable pain should the knife stab it. In this paper we consider what happens when a person's real body is visually substituted by a lifesized virtual body, and they see a threat or attack to a hand of this virtual body. Our experiment investigates brain activity in response to events that would cause pain to the observer were these 'pain observation' events to occur in reality. Our contribution lies in introducing a new technique for the study of such pain observation, by using immersive virtual reality (IVR) for the scenario and stimulation, while recording brain activity with EEG. Our work contributes to the growing field of body representation, how the brain represents the body, as well as presenting results on pain observation.

Several brain imaging techniques have used pain observation experiments to understand the associated mental processes. Methods that employ Magnetic Resonance Imaging (fMRI) have found that the anterior cingulate cortex and the right insula brain regions are associated with such pain observation (Jackson et al., 2005, Gu and Han, 2007). Studies that have examined the Event Related Potential (ERP) temporal dynamics involved in empathy, measured as the response to observation of pain in others, especially prominent in the motor cortex area, have found greater P450 responses for painful images compared to neutral images (Fan and Han, 2008, Li and Han, 2010, Meng et al., 2012, Meng et al., 2013). These effects were modulated by the realism of the presentation and were stronger with greater realism (Fan and Han, 2008). Similarly, studies using Transcranial Magnetic Stimulation (TMS), participants have shown a reduction in Motor Evoked Potentials (MEPs) resulting from watching 
a hand undergoing a painful situation (Avenanti et al., 2005). Experiments combining both pain observation and electrical stimulation have shown modulations in the somatosensory evoked potentials (SEP), particularly prominent in the centroparietal locations, with larger amplitudes for the P450 component when observing a painful situation (Bufalari et al., 2007).

This automatic empathy response is elicited involuntarily (Preston and de Waal, 2002) through a bottom-up process. However it can also be modulated consciously (top-down), for example under instructions of subjective pain estimation, generating stronger P450 responses (Fan and Han, 2008).

Interestingly, pain observation studies that have focused on Frequency Power Spectra (FPS) decomposition have shown a depression in the mu-rhythm during painful conditions, using magnetoencephalography (MEG) and EEG (Cheng et al., 2008, Yang et al., 2009). This abolition or suppression of the mu-rhythm when observing painful situations has been interpreted to be in agreement with previous observations about the involvement of this oscillatory activity in the execution of voluntary movements (Neuper et al., 2005). The mu-ERD is described as a circumscribed desynchronization in the upper alpha frequency band (in the range of about 9-12Hz) when a participant performs a motor action (Pfurtscheller and Lopes da Silva, 1999) or motor action observation (Muthukumaraswamy and Johnson, 2004). Moreover, previous studies have found that mu-ERD can also be triggered as an unconscious mechanism to avoid painful events (Babiloni et al., 2008). When a sound alerted participants 2.5 seconds prior to an electrical painful stimulation at the left index finger, a suppression of the mu-rhythm was elicited, as if the participant had tried to move the hand to avoid harm. This effect was not elicited during non-painful stimulation. 
We present a pain observation experiment in the context of a whole body ownership illusion in immersive virtual reality (IVR). The IVR was delivered through a wide field-of-view head-tracked stereo head-mounted display (HMD). This setup substitutes a person's own body by a virtual body seen from a first person perspective (1PP), such that when participants look down towards their body they would see a virtual body replacing their own (Figure 1). In the experiment the participant's stationary right virtual hand, which was collocated with the real right hand resting on a table, was repeatedly threatened by a virtual knife, thereby reproducing in IVR previously conducted pain observation experiments (Avenanti et al., 2006, Bufalari et al., 2007, Gu and Han, 2007, Fan and Han, 2008, Li and Han, 2010, Meng et al., 2012, Meng et al., 2013). The painful stimulation was compared to a control where the same knife attacked only the virtual table that was spatially registered with the real table on which the hand was resting (Figure 1). In short we measured EEG responses, which resulted in similar ERPs compared to previous experiments, with greater P450 effects in CP3 for the painful condition compared to the control condition (Bufalari et al., 2007, Fan and Han, 2008, Li and Han, 2010, Meng et al., 2013).

We used IVR for the study of pain observation due to recent results that show that virtual reality can be used to induce an illusion of ownership over a virtual body. This work has its origin in the rubber hand illusion, where it has been shown that synchronous tactile stimulation of a visible rubber hand and the experimental subject's corresponding hidden real hand, results in an illusion of ownership over the rubber hand (Botvinick and Cohen, 1998, Armel and Ramachandran, 2003, Ehrsson et al., 2004, Tsakiris and Haggard, 2005). Here the rubber hand is placed on a table in front of the subject in an anatomically plausible position, with the corresponding real 
hand out of sight behind a screen. When the real and rubber hand are synchronously brushed in the same location on each hand then there is typically and quickly an illusion of ownership over the rubber hand. This result has been extended to a virtual hand in virtual reality (Slater et al., 2008) including, but less strongly, a table-top video projection of a hand (Ijsselsteijn et al., 2006), and the illusion is also reproduced when visuomotor synchrony is used rather than visuotactile (Sanchez-Vives et al., 2010, Kalckert and Ehrsson, 2012).

Similar multisensory techniques have been used for whole body ownership illusions both illusions of displacement (or out of the body illusions) (Ehrsson, 2007, Lenggenhager et al., 2007), and illusions of body substitution (Petkova and Ehrsson, 2008). Evidence suggests that the dominant factor in such whole body illusions may be first person perspective (Slater et al., 2010, Petkova et al., 2011, Maselli and Slater, 2013), though it is likely that additional multisensory stimulation such as visuotactile and visuomotor synchrony would also play a role. For a review of the field see (Blanke, 2012).

Typically, however, pain observation experiments present a series of pictures with hands or other extremities undergoing painful situations, and they compare the brain response of the participants to the activation produced by pictures where the same extremities do not undergo painful situations (Avenanti et al., 2006, Bufalari et al., 2007, Fan and Han, 2008, Li and Han, 2010). Many of these experiments present scissors and needles perforating the extremities as painful stimuli. A potential advantage of IVR, however, is that there is greater ecological validity, going beyond the presentation of two-dimensional, static stimuli. With IVR there is a life-sized, three dimensional virtual body seen in stereo, that visually substitutes the obscured real body of the participant, which can be virtually attacked. Hence the level of 
realism can be greatly enhanced. In the present study participants saw a knife attacking the hand of their virtual body, the virtual body therefore acting as a surrogate for the real body in the context of pain observation.

\section{Materials and methods}

\subsection{Apparatus}

Participants were fitted with a stereo NVIS nVisor SX111 head-mounted display (HMD). This has dual SXGA displays with $76^{\circ} \mathrm{H} \times 64^{\circ} \mathrm{V}$ degrees field of view (FOV) per eye, totaling a wide field-of-view $111^{\circ}$ horizontal and $60^{\circ}$ vertical, with a resolution of $1280 \times 1024$ per eye displayed at $60 \mathrm{~Hz}$. Head tracking was by a $6-\mathrm{DOF}$ Intersense IS-900 device. The virtual environment was programmed in the XVR system (Tecchia et al., 2010) and the virtual character rendered using the HALCA library (Gillies and Spanlang, 2010).

\subsection{Procedures}

Nineteen healthy volunteers - 9 male, 10 female; aged $25 \pm 4.0$ (S.D.) years - all righthanded - participated in the experiment. The experimental protocol was approved by the Universitat de Barcelona Ethics Committee (Spain), and all the participants gave written informed consent and were paid $10 €$ for their participation.

Participants entered the virtual reality, and saw a virtual body (avatar) from 1PP that was consistent with their gender and skin color. The virtual scene consisted of the avatar seated on a chair with its virtual right hand placed on a desk. In the laboratory, the participant was seated with his/her real right hand collocated with the avatar's hand and resting on a table. The left hand was placed comfortably on the participant's 
lap. Participants were asked to relax and keep their arms and hand still throughout the experiment (Figure 1).

Participants were encouraged to freely look around for 60 seconds to familiarize themselves with the environment while keeping their arms still and collocated with those of the virtual body. After the familiarization time, we told participants several times to fix their gaze on the virtual hand resting on the table and to keep their real hand still. We did not ask them to perform any other task at all, such as pain judgment, but only to fixate on the virtual hand.

\subsection{Stimuli}

Participants repeatedly experienced two conditions in a within-group design: condition HAND where the knife stabbed the virtual right hand, and condition TABLE where the knife stabbed the table, $15 \mathrm{~cm}$ away from the right hand (Figure 1). The experiment consisted of 70 trials repeating the HAND and TABLE conditions (30 HAND and 40 TABLE). A trial consisted of three parts:

1. Pre-stimulus: the participant looked at the virtual hand (5-8 seconds).

2. Stimulus: a knife appeared in the HAND or TABLE (2 seconds).

3. Black screen: a black screen appeared (2 seconds).

During the first 10 trials only the TABLE condition was presented to acclimatize participants to the trial evolution and the black screen. Then, there were 6 predefined blocks of 10 trials each, each block had 5 HAND and 5 TABLE, with the order randomized within each block. The order in which these blocks were presented to the participants was randomized for each participant. After the 70 trials the screen went black and the experiment ended. This virtual reality exposure lasted for 15 minutes. 
(See Electronic Supplementary Material Video for an overview of the whole experiment). Participants then completed a questionnaire about their virtual experience, in which they answered the following questions:

1. Ownership: I felt as if the hand I saw in the virtual world might be my hand.

2. Harm Hand: I had the feeling that I might be harmed when I saw the knife inside the hand.

3. Harm Table: I had the feeling that I might be harmed when I saw the knife outside the hand.

4. No Ownership: The hand I saw was the hand of another person.

5. Body Threat: I saw the knife as a threat to my body.

Responses to these statements were on a 5-point Likert-scale where 1 was anchored to strong disagreement and 5 to strong agreement. Questions 1 and 4 were related to the sense of ownership of the hand, with question 1 expected to record high scores while question 4 expected to record low scores. These two questions are similar to those used in previous studies to measure ownership illusions (Banakou et al., 2013, Llobera et al., 2013) for example: "How much did you feel that the virtual body was your body" for the ownership question, and "How much did you feel that the virtual body was another person?" as a control for the no ownership, or "How much did you feel that the virtual body you saw when you looked down at yourself was your own body", versus "How much did you feel as if you had two bodies". Moreover question 1 is similar to that used by the original Botvinick and Cohen (1998) paper "I felt as if the rubber hand were my hand". Questions 2 and 3 were to examine whether there was any feeling of harm in response to the knife being in the condition HAND or 
TABLE. Question 5 was a consistency check to control questions 2 and 3; we expect similar responses to Harm Hand.

\subsection{Electrophysiological Recording}

Both EEG and electromyography (EMG) were recorded using an gUSBamp ${ }^{1}$ amplifier with a resolution of $30 \mathrm{nV}$; the electrodes were set to cover the motor cortex area and surrounding: FC3, FC4, C3, C4, CP3, CP4 located according to the 10/20 standard EEG recording; the reference was set with an ear clip on the left ear lobe; the ground was positioned on the forehead; electrodes in the face measured ocular activity (EOG). Three EMG electrodes were placed in the flexor carpi ulinaris muscle of the right arm to measure whether participants moved their hand. All the electrodes were kept to impedances below $10 \mathrm{k} \Omega$. The data was recorded using Matlab with a sampling frequency of $512 \mathrm{~Hz}$. Trials that were contaminated, i.e., exceeding amplitudes of $\pm 100 \mu \mathrm{V}$ by any electrode, or by the EOG were rejected off-line; $3.8 \%$ of the trials were excluded due to artifacts $(2.26 \pm 2.42$ trials per participant $)$.

\subsection{EEG Data analys is}

In order to study the effects of the stimuli on the pain sensitivity, Event Related Potential (ERP) components were analyzed as in (Fan and Han, 2008, Li and Han, 2010, Meng et al., 2012). The stimulus locked ERP helped us determine the pain related levels of the participants with respect to the HAND condition, where a higher P450 activity was expected in case of a pain response.

The ERPs in both conditions HAND and TABLE were averaged separately for each subject. The ERPs were also used to better study the lateralization part of the

\footnotetext{
${ }^{1}$ The EEG equipment was supplied by Guger Technologies, www.gtec.at
} 
Readiness Potential in order to detect which hemisphere was more active. The Readiness Potential has been previously related to movement preparation and it is generally calculated as the double subtraction of C3 - C4 (Eimer, 1998), considering right and left hand movements. As in our case we only used right hand manipulations we report only one side $\mathrm{C} 3$ - $\mathrm{C} 4$ subtraction. An increase in negativity is expected when a movement is prepared with the contra lateral hemisphere. Thus, negative amplitude might reflect a right hand movement preparation.

Apart from the ERPs, frequency bands were also evaluated. To account for variations we used short-time power spectra as described in (Pfurtscheller and Lopes da Silva, 1999). Power Spectral Density (PSD) was calculated as the superimposed 1-s power spectra calculated over the event-related EEG for the HAND and TABLE conditions for both the reference and activity periods.

\subsection{EMG Data analys is}

EMG data was filtered with a band pass of 20 to $250 \mathrm{~Hz}$ selected according to the recommendations of (Fridlund and Cacioppo, 1986) and keeping the frequency range where the primary energy in the surface EMG signal is located. As is common practice the Root Mean Squared (RMS) processing technique was used (Fridlund and Cacioppo, 1986). The RMS of the signal was computed with a sliding window of 500 $\mathrm{ms}$ in order to detect if right arm muscles were activated at any moment. For the purpose of this experiment subjects were asked not to move their hand under any circumstance, and the plan was that trials showing EMG activation would be discarded. 


\section{Results}

\subsection{Hand Movements}

A critical question for this experiment was whether participants did actually move their threatened hand or not. Real hand movement had to be negligible otherwise it would increase activation in the motor cortex. This was assessed using the EMG data. The RMS was calculated for the pre-stimuli reference period (-1 to 0 seconds) and the post-stimuli activation period ( 0.7 to 1.7 seconds), these periods correspond to the time when the motor cortex was found activated. Using a repeated measures ANOVA comparing (HAND-BASELINE vs. TABLE-BASELINE) no significant difference nor effects were found in the RMS, $F(1,18)=2.685, \mathrm{P}=0.119$. Other timings also did not show any activation, and the same ANOVA analysis was later used to analyze the mu-ERD. These results suggest that the participants did not move their real hand during the experimental period (see Discussion where this issue is revisited).

\subsection{Questionnaire}

Here we consider whether the setup did induce an illusion of ownership over the virtual hand, and whether the stabbing knife was subjectively experienced as a threat. Figure 2 shows the box plot $(n=19)$ for the questionnaire responses that were designed to assess this. It is clear that the illusion of ownership was high (the median level of ownership is 5), and the no ownership score was comparatively low (the median is 2). The Wilcoxon matched pairs sign-rank test (two-sided) comparing Ownership with No Ownership shows that this difference is significant $(\mathrm{z}=3.89, \mathrm{P}<$ 0.0001). The illusion of harm to the hand (Harm Hand) has median 3, and Harm Table has median 1 . The paired sign rank test again shows these to be significantly 
different $(\mathrm{z}=3.74, \mathrm{P}<0.0002)$. The threat to the body as a whole (Body Threat) also has median 3, and is significantly different from Harm Table $(\mathrm{z}=3.59, \mathrm{P}<0.0003)$. Although the medians of Harm Hand and Body Threat are the same, the greater range of the former leads to it being significantly greater overall $(\mathrm{z}=2.36, \mathrm{P}<0.018)$.

Table 1 shows that Ownership is positively correlated with Harm Hand which is positively correlated with Body Threat. Body Threat is also positively correlated with Ownership. There are no other significant correlations. This is important since illusory ownership of the hand should go along with the feeling of threat to that hand or to the body, since without illusory ownership there is no actual threat. These results are consistent with the original hypothesis that the stronger the illusion of ownership the greater the tendency of participants to give higher ratings to the Harm questions. We consider these relationships in greater depth in Section 3.6.

\subsection{ERP Stimulus-locked activity}

The pain sensitivity levels of the participants for the HAND and TABLE conditions were assessed using stimulus-locked ERPs depicted in Figure 3. A repeated measures ANOVA P450 [condition (HAND/TABLE) · electrode (Frontal/Central/Centroparietal) $\cdot$ hemisphere (Left, Right)] in the time window 420 to $620 \mathrm{~ms}$ on the original real voltage data showed a significant main within subjects effect for Condition $(\mathrm{F}(2,18)=6.977, \mathrm{P}=0.017)$ and for Electrode position $(\mathrm{F}(2,36)=21.401, \mathrm{P}<0.001) . \mathrm{A}$ centroparietal distribution was observed for the P450 component as reflected by the significant interaction between Condition and Electrode $(\mathrm{F}(2,36)=7.640, \mathrm{P}=0.002)$ (the peak value was observed at CP3, see Figure 3). We conducted further post-hoc pairwise comparisons between both conditions (hand and table) at parietal and central electrodes; the paired samples t-test were significant for the P450 at C3 and CP3 
electrodes $(\mathrm{t}(18)=3.438, \mathrm{P}=0.003$ and $\mathrm{t}(18)=3.637, \mathrm{P}=0.002$, respectively). These results are consistent with the $\mathrm{P} 450$ effects induced when a pain estimation task was performed in previous studies (Fan and Han, 2008, Li and Han, 2010, Meng et al., 2012).

\subsection{Frequency Power Spectral Density}

To determine whether participants showed a different frequency response to the attack (HAND) versus the control stimulus (TABLE), we performed a 1-s power spectra analysis (see Figure 4abc). In Figure 4a the Time Frequency evolution of the two conditions and the difference in the spectral activity can be observed; further representation of the Mu-rhythm evolution can be found in Figure 4c; and the 1-s power spectral differences between the reaction ( 0.7 to 1.7 seconds) and the baseline (-1 to 0 seconds) can be found in Figure 4b. The three visualizations show a clear attenuation in the mu-rhythm during the HAND condition.

The 1-s power spectrum of the mu-rhythm $(9-12 \mathrm{~Hz})$ in both conditions (hand-baseline vs. table-baseline) was used for the statistical analysis. A repeated measures ANOVA with three factors [condition (HAND/TABLE) · electrode (Frontal/Central/Centroparietal) · hemisphere (Left, Right)] was run to analyze the desynchronization. We found a significant main within-subject effect for the condition $(F(1,18)=12.235$, $\mathrm{P}=0.003)$. The distribution of this component was dependent on the electrode position as reflected by the significant interaction (condition $\cdot$ electrode $F(2,36)=8.751, P$ $=0.001)$. Further post-hoc tests comparing the conditions in the parietal and central electrodes showed most prominent desynchronizations during the HAND condition in $\mathrm{C} 3(\mathrm{t}(18)=-3.482, \mathrm{P}=0.003)$ and $\mathrm{CP} 3(\mathrm{t}(18)=-3.670, \mathrm{P}=0.002)$. These results are 
similar to the mu-ERD effects induced when an imaginary hand movement is performed (Pfurtscheller and Lopes da Silva, 1999, Neuper et al., 2005).

\subsection{Readiness Potential}

To detect which hemisphere was more activated, and thus if there was any movement preparation (Eimer, 1998), we calculated the Readiness Potential as C3 - C4. An increase in negativity is expected if a movement is prepared with the contra lateral hemisphere.

Figure 4d depicts the response-locked Readiness Potential (C3-C4) activity that was analyzed via a paired-samples t-test for time-window 300 to $500 \mathrm{~ms}$. A significant difference between conditions was found $(\mathrm{t}(19)=-2.237, \mathrm{p}=0.038)$. This result shows negativity in the contralateral hemisphere (left, $\mathrm{C} 3$ electrode) during the HAND condition (Mean=-0.455 Std=1.183), which indicates right hand pre-movement activity versus a more positive response during the TABLE condition (Mean $=0.419$ $\mathrm{Std}=1.221)$.

\subsection{Relationship Between Questionnaire Scores, P450 and mu}

Here we examine the relationship between the EEG response variables (P450, mu), the condition (TABLE, HAND), and the subjective responses from the questionnaire. Table 2 shows strong positive correlations between Ownership and each of P450 and mu, and a positive correlation between Harm Hand and P450. There is a negative correlation between No Ownership and mu.

Correlations do not imply causality, but the fact that there are very strong correlations between variables obtained in totally different ways (questionnaire and electrical recordings from the scalp) suggests that there is something to be explained. It would 
be surprising indeed if these were just coincidental, especially given the underlying supposition of this paper that the level of ownership would be reflected in brain activity in just the way that these correlations suggest. In particular given the setup and based on previous results showing that body ownership is likely to be induced as a result of 1PP (Slater et al., 2010), we would expect that the level of ownership would be positively associated with the feelings of threat to the hand and the body, which in turn would influence the P450 and mu values. These would also be influenced by the manipulated condition (i.e., whether the knife penetrated the hand or was close to it but did not penetrate).

Conventional approaches would have to treat these different relationships in separate linear models (for example, using regression) that cannot assess multiple simultaneous effects. To address this we turned to path analysis - for example (Kaplan, 2009) - which can model multiple simultaneous stochastic equations. Although not conventional in this domain of research it is a powerful method that we have used before in the context of body ownership studies (Kilteni et al., 2013, Llobera et al., 2013, Maselli and Slater, 2013, Steptoe et al., 2013).

Path analysis is particularly appropriate in the case when there are several strong correlations between variables, and a hypothesized model specifying potential causal relationships amongst them. The model is expressed as a set of stochastic equations (not necessarily linear) with the dependent variables on the left hand sides and the functional specifications of the model relationships on the right (plus random error). Path analysis estimates the resulting covariance matrix of this model (and the parameters involved in the equations) typically using maximum likelihood estimation. It can unravel spurious correlations, for example, when $\mathrm{x}$ and $\mathrm{y}$ are apparently highly correlated but where actually they are each influenced separately by another variable 
$z$. A model that consisted of $(z \rightarrow x, z \rightarrow y, x \rightarrow y)$ where ' $u \rightarrow$ ' represents a potential causal relationship from $u$ to $w($ e.g. an equation of the form $w=\alpha+\beta u+\varepsilon$ ) would find that the path $\mathrm{x} \rightarrow \mathrm{y}$ was not significant (in spite of a high correlation between $\mathrm{x}$ and y). We used path analysis to try to isolate potential causal paths from correlations. For the path analyses we used Maximum Likelihood estimation, with robust standard errors, available in Stata 13 (www.stata.com), and the questionnaire responses were modeled as ordinal logistic variables.

We fitted the model allowing Ownership to influence Harm Hand, Harm Table and Body Threat. In turn these could influence P450 and mu, which were also influenced by condition. We fitted the path model and deleted paths with significance levels less than 0.05 . The resulting path model is shown in Figure 5 and associated Table 3. It can be seen that Ownership is very strongly positively associated with the three harm variables. Harm Hand is very strongly positively associated with P450 and weakly with mu. Harm Table is weakly negatively associated with P450. Condition is strongly positively associated with P450 and negatively with mu. The overall fit of the model is good as shown by the last column of Table 3 which presents the correlations between values fitted by the model and the observed values of the response variables $\mathrm{P} 450$ and $\mathrm{mu}$.

Now turning attention to the Readiness Potential (RP), this is based on a different set of data $(\mathrm{n}=38)$ since RP is a bipolar difference of the activity between the C3 and $\mathrm{C} 4$ electrodes in the motor cortex so cannot be considered at the same time as P450 and mu. Applying path analysis to this data, only condition and Harm Table are significantly related to RP. Hence an ordinary regression can be used (although still we allow robust standard errors). The result is shown in Table 4, where condition 
(Knife in Hand) is negatively associated with RP but positively associated with Harm Table. This is consistent with a lateralization between hemispheres occurring during the preparation of a motor action, the $\mathrm{RP}(\mathrm{C} 3-\mathrm{C} 4)$ is more negative when there is preparation to move the right hand (Eimer, 1998), which in the current experiment is the attacked hand. Therefore, a reduction in RP for higher scores in Harm Hand question indicates stronger preparation of movement.

\section{Discussion}

Our results suggest that participants instinctively avoided a virtual knife stab to their virtual body, thus activating the motor cortex and generating a mu-ERD, and a Readiness Potential, as would be expected if their real hand were threatened. Our study reproduced the results of (Fan and Han, 2008, Li and Han, 2010, Meng et al., 2012, Meng et al., 2013) in terms of ERP correlates, showing significant evidence that pain effects were found, with the mean P450 showing greater amplitudes at the CP3 electrode location in the HAND compared to the TABLE condition.

Importantly, participants had been instructed not to move their hand during the whole experiment - and this was verified by the EMG analysis. However, it is important to note that measurements of the flexor carpi alone could not have detected very subtle movements, a reason for caution. Nevertheless, when doing the ERP study we found motor cortex activation in the HAND condition with a significantly greater negative Readiness Potential (C3-C4), associated with the intention of moving the right hand, and this Readiness Potential was probably an instinctive reaction to the harm that could not be controlled consciously by the participants. 
Additionally, we found that when the virtual hand was attacked with the virtual knife, it elicited significant motor cortex activation. A significant mu-ERD was found when the knife attacked the hand - especially prominent in the $\mathrm{C} 3$ electrode - as if the participant were trying to avoid harm. This suppression of the mu-rhythm in the HAND condition could be interpreted as being in agreement with previous observations about the involvement of this oscillatory desynchronization when a participant performs a hand motor action (Pfurtscheller and Lopes da Silva, 1999). Besides, this effect reproduces the results of (Yang et al., 2009, Perry et al., 2010, Whitmarsh et al., 2011), although we believe that the illusory feeling of ownership over the virtual body was likely much greater than in any previous pain observation experiment. Furthermore, this illusory ownership provoked more prominent responses with greater similarity to those described by (Babiloni et al., 2008) in preparation for an electrical painful stimulation of the left index finger.

A recent paper (Evans and Blanke, 2013) showed that synchronous visual-tactile feedback during the hand ownership illusion generates mu-ERD in the sensorimotor cortex similar to the one produced during motor imagery BCI. Although, in our experiment no tactile feedback was provided, we postulate that their results are compatible with our findings and suggest that the correlations found in the current experiment between the mu-ERD and P450 with the ownership illusion question may be related by a similar mechanism to the one they describe. Future research could assess whether tactile feedback would enhance the experience in the current scenario and inhibit any existing sensory mismatch. In our study tactile feedback was avoided to prevent overlaying activities in the sensorimotor cortex between the interpretation of tactile sensory information and the efferent motor reactions (Yetkin et al., 1995). It would have been very difficult to dissociate the effects of the tactile stimulation from 
the subconscious motor reaction to the harm. However, regarding the sensory mismatch, some participants reported a strange feeling in their finger at the end of the experiment that would indicate that they were having illusions of tactile stimulation through a top down mechanism.

According to (Pfurtscheller and Lopes da Silva, 1999) an ERS in the beta-rhythm would typically be found in hand motor-imagery when the movement finishes. Nevertheless, in the current experiment we could not find significant beta rebound.

We have shown that automatic neural mechanisms, such as pain responses, that occur in reality occurred in this case in response to events in the virtual reality scenario of this study. This is in line with previous findings that people do tend to have similar responses in IVR as they would to similar situations in reality (Sanchez-Vives and Slater, 2005), and this study seems to confirm this at the level of brain activity as measured by EEG.

Additionally the results are useful for understanding the neural and cognitive mechanisms of body perception. We have shown that neural responses (P450, mu and RP) are correlated with the subjective level of the ownership illusion and the subjective illusions of harm and threat to the body. It seems quite remarkable that these variables, being in principle totally unrelated to one another (electrical brain signals measured from the scalp compared with scores in a questionnaire) are nevertheless apparently strongly related. This correlation provides a cross validation that both the questionnaire responses and the electrical signals relate to the same underlying brain processes. However, correlations should not be confused with causation, and the path analysis proved useful for investigating a causal model between the observed variables. For example, although there is a positive correlation 
between Body Threat and P450 (Table 2), which might simplistically be interpreted as a direct causal relation, in the context of the path model this can be seen as spurious. The model provides an alternative interpretation that Ownership is positively associated with Harm Hand, which in turn is positively associated with P450. But also Ownership is positively associated with Body Threat. Overall the path analysis was able to unravel possible relationships that would otherwise not be apparent, and provide a quantitative assessment of a model.

From the path model P450 is higher in the HAND compared to the TABLE condition, and it is also higher the stronger the subjective feeling that the hand might be harmed. But whose hand? A possible caveat in the interpretation of the results is that we cannot easily dissociate some of the intrinsic factors that may be modulating the pattern of ERP responses observed, for example, between empathy and body ownership. Previous empathy related studies (Fan and Han, 2008, Li and Han, 2010, Meng et al., 2012, Meng et al., 2013) suggest that the P450 component is mostly associated to empathy processing. Here, however, it appears to be related to ownership, given that Harm Hand specifically refers to harm to the participant (“...I might be harmed ...") a statement that is not compatible with an interpretation that only favours empathy.

Empathy refers to the capacity to respond and understand experiences of another person (Decety and Jackson, 2004). Brain activity associated with empathic responses occurs, for example, in the context of pain observation of the (even violet coloured) hand of a stranger (Avenanti et al., 2010). However, the same study shows that it is not generated when the hand belongs to racial outgroup members (specifically white individuals observing black hands). However, recent evidence suggests that ownership by white individuals over a black rubber arm reduces implicit racial bias 
(Maister et al., 2013) as does ownership of a dark skinned virtual body (Peck et al., 2013). Since (Avenanti et al., 2010) found that the degree of implicit racial bias and empathy responses were negatively correlated, we could conclude that embodiment in the body of another might be likely to increase empathy towards that person or the stereotype or group that the person represents (other things being equal). So although empathy and body ownership are not the same, they are related - for body ownership may be used to manipulate the degree of empathy.

Perhaps the common factor between empathy and body ownership is perspective taking (Lamm et al., 2007). Perspective taking denotes the ability to see the world from the eyes of another, and metaphorically to put yourself in the shoes of another. It has been shown, for example, that perspective taking can improve attitudes towards others such as racial or ethnic outgroups (Swart et al., 2010). However, virtual embodiment provides a technological method for actually realising perspective taking - when embodied in a virtual body it is literally the case that you see through the eyes of a (virtual) other, so it is not especially surprising that virtual embodiment can lead to a change in empathy, since it well realises perspective taking. However, we would argue that in the present study empathy plays less of a role - except in the tautological sense that you might have 'maximal' empathy towards yourself, your own body. We suggest that this 'maximal' level of empathy may be a reason why stronger reactions were found in the motor cortex in our experiment in comparison to previous pain observation studies.

In our study we observed new effects (mu-ERD and lateralization) that have not been reported before in previous empathy studies. We believe that these strong effects were observed due to the strong embodiment illusion. Previous research has shown that embodiment can be modulated by different combinations of self-location and body 
ownership (Longo et al., 2008, Kilteni et al., 2012). In our setup the control condition TABLE in which the knife did not appear where the hand was located, but $15 \mathrm{~cm}$ away, did not trigger the brain activation, indicating therefore that the possibility of harm to the own body played an important role. Our results show that the exploitation of virtual body ownership illusions could be useful for further understanding the underlying neural mechanisms involved in cognitive processes of perception. Besides, the measurements of cognitive processes provide a promising tool to measure virtual embodiment.

This may also have implications not only for the measurement of virtual body ownership but also to discriminate the strength of this illusion, so that people reacting with a stronger EEG activation - greater P450 amplitude the virtual hand is attacked may have a stronger illusion than people with weaker P450 amplitude. This is indicated in the path diagram (Figure 5) where the subjective level of ownership is seen to be indirectly associated with both P450 and mu.

The questionnaire responses indicated generally a very strong illusion of ownership over the virtual body. This could explain why the brain responses observed - P450, Readiness Potential and mu-ERD - were larger in comparison to previous experiments reported in the literature as observed above. A future experiment could explicitly test this by reducing the level of ownership through a third person perspective rather than a first person perspective condition (Slater et al., 2010, Petkova et al., 2011, Peck et al., 2013). For example, this would involve observing the reactions to seeing somebody else being attacked in an immersive virtual environment. These results could also be further studied by focusing on the effects of self-location with respect to the threat-stimuli. In general the neural responses by themselves may provide a non-subjective measure of embodiment, however the 
current findings are based on correlations of both objective and subjective measures. Further studies may explore the extent in which ERPs may be exploited as an objective measure of embodiment.

\section{Conclusions}

The present study suggests that, when a person is in an immersive virtual reality, and has body ownership illusion towards a virtual body that apparently substitutes their own body, there are autonomic responses that correspond to what would be observed were the events to take place in reality. Overall automatic brain mechanisms $-\mathrm{P} 450$ were found in this variation of the classical pain observation experiment, which is consistent with what (Bufalari et al., 2007, Fan and Han, 2008, Li and Han, 2010, Meng et al., 2012, Meng et al., 2013) previously reported. However, our setup was not one concerned with participants experiencing empathy towards another person but rather experienced direct attacks to their own body, since both subjective and objective data point in that direction. The results support our initial hypothesis that a threat to a virtual hand, towards which the participant has an illusion of ownership, would significantly produce a harm prevention effect (measures using the Readiness Potential (C3-C4) and oscillatory movement-related components, the mu-ERD), such as trying to move it away from the source of the harm. The questionnaire also confirmed high levels of ownership over the virtual body (see Figure 2). In addition, the correlation between the automatic brain mechanisms $-\mathrm{P} 450$ - and the subjective illusion of ownership opens the door for a new promising measure of virtual embodiment. 


\section{Acknowledgements}

This study was funded by the European Union FP7 Integrated Project VERE (No.

257695). MGF's research was supported by the FI-DGR predoctorate grant from the

Catalan Government co-founded by the European Social Found (EC-ESF). ARF has

been supported by a research grant from the Spanish government (PSI2011-29219).

The ERC project TRAVERSE (\#227985) also contributed towards this research.

\section{References}

Armel KC, Ramachandran VS (2003) Projecting sensations to external objects: evidence from skin conductance response. Proceedings Biological sciences / The Royal Society 270:1499-1506.

Avenanti A, Bueti D, Galati G, Aglioti SM (2005) Transcranial magnetic stimulation highlights the sensorimotor side of empathy for pain. Nature neuroscience 8:955-960.

Avenanti A, Paluello IM, Bufalari I, Aglioti SM (2006) Stimulus-driven modulation of motor-evoked potentials during observation of others' pain. Neuroimage 32:316-324.

Avenanti A, Sirigu A, Aglioti SM (2010) Racial Bias Reduces Empathic Sensorimotor Resonance with Other-Race Pain. Current biology : CB 20:1018-1022.

Babiloni C, Capotosto P, Brancucci A, Del Percio C, Petrini L, Buttiglione M, Cibelli G, Romani GL, Rossini PM, Arendt-Nielsen L (2008) Cortical Alpha Rhythms Are Related to the Anticipation of Sensorimotor Interaction Between Painful Stimuli and Movements: A High-Resolution EEG Study. The journal of pain : official journal of the American Pain Society 9:902-911.

Banakou D, Groten R, Slater M (2013) Illusory ownership of a virtual child body causes overestimation of object sizes and implicit attitude changes. Proceedings of the National Academy of Sciences.

Blanke O (2012) Multisensory brain mechanisms of bodily self-consciousness. Nature Reviews Neuroscience 13:556-571.

Botvinick M, Cohen J (1998) Rubber hands' feel'touch that eyes see. Nature 391:756756.

Bufalari I, Aprile T, Avenanti A, Di Russo F, Aglioti SM (2007) Empathy for Pain and Touch in the Human Somatosensory Cortex. Cerebral Cortex 17:25532561.

Cheng Y, Yang C-Y, Lin C-P, Lee P-L, Decety J (2008) The perception of pain in others suppresses somatosensory oscillations: A magnetoencephalography study. Neuroimage 40:1833-1840.

Decety J, Jackson PL (2004) The functional architecture of human empathy. Behavioral and cognitive neuroscience reviews 3:71-100. 
Ehrsson HH (2007) The Experimental Induction of Out-of-Body Experiences.

Science 317:1048.

Ehrsson HH, Spence C, Passingham RE (2004) That's My Hand! Activity in Premotor Cortex Reflects Feeling of Ownership of a Limb. Science 305:875-877.

Eimer M (1998) The lateralized readiness potential as an on-line measure of central response activation processes. Behavior Research Methods, Instruments, \& Computers 30:146-156.

Evans N, Blanke O (2013) Shared electrophysiology mechanisms of body ownership and motor imagery. Neuroimage 64:216-228.

Fan Y, Han S (2008) Temporal dynamic of neural mechanisms involved in empathy for pain: An event-related brain potential study. Neuropsychologia 46:160173.

Fridlund AJ, Cacioppo JT (1986) Guidelines for human electromyographic research. Psychophysiology 23:567-589.

Gillies M, Spanlang B (2010) Comparing and evaluating real time character engines for virtual environments. Presence: Teleoperators and Virtual Environments 19:95-117.

$\mathrm{Gu}$ X, Han S (2007) Attention and reality constraints on the neural processes of empathy for pain. NeuroImage 36:256-267.

Ijsselsteijn WA, de Kort YAW, Haans A (2006) Is This My Hand I See Before Me? The Rubber Hand Illusion in Reality, Virtual Reality, and Mixed Reality. Presence: Teleoperators and Virtual Environments 15:455-464.

Jackson PL, Meltzoff AN, Decety J (2005) How do we perceive the pain of others? A window into the neural processes involved in empathy. Neuroimage 24:771779 .

Kalckert A, Ehrsson HH (2012) Moving a rubber hand that feels like your own: dissociation of ownership and agency. Frontiers in Human Neuroscience 6.

Kaplan D (2009) Structural Equation Modeling: Foundations and Extensions: SAGE Publications.

Kilteni K, Bergstrom I, Slater M (2013) Drumming in immersive virtual reality: the body shapes the way we play. Visualization and Computer Graphics, IEEE Transactions on 19:597-605.

Kilteni K, Normand J-M, Sanchez-Vives MV, Slater M (2012) Extending body space in immersive virtual reality: a very long arm illusion. PloS one 7:e40867.

Lamm C, Batson CD, Decety J (2007) The neural substrate of human empathy: effects of perspective-taking and cognitive appraisal. Journal of Cognitive Neuroscience 19:42-58.

Lenggenhager B, Tadi T, Metzinger T, Blanke O (2007) Video Ergo Sum: Manipulating Bodily Self-Consciousness. Science 317:1096-1099.

Li W, Han S (2010) Perspective taking modulates event-related potentials to perceived pain. Neuroscience Letters 469:328-332.

Llobera J, Sanchez-Vives MV, Slater M (2013) The relationship between virtual body ownership and temperature sensitivity. Journal of The Royal Society Interface 10.

Longo MR, Schüür F, Kammers MPM, Tsakiris M, Haggard P (2008) What is embodiment? A psychometric approach. Cognition 107:978-998.

Maister L, Sebanz N, Knoblich Gn, Tsakiris M (2013) Experiencing ownership over a dark-skinned body reduces implicit racial bias. Cognition 128:170-178.

Maselli A, Slater M (2013) The building blocks of the full body ownership illusion. Frontiers in Human Neuroscience 7. 
Meng J, Hu L, Shen L, Yang Z, Chen H, Huang X, Jackson T (2012) Emotional primes modulate the responses to others' pain: an ERP study. Exp Brain Res 220:277-286.

Meng J, Jackson T, Chen H, Hu L, Yang Z, Su Y, Huang X (2013) Pain perception in the self and observation of others: An ERP investigation. NeuroImage 72:164173.

Muthukumaraswamy SD, Johnson BW (2004) Primary motor cortex activation during action observation revealed by wavelet analysis of the EEG. Clinical neurophysiology : official journal of the International Federation of Clinical Neurophysiology 115:1760-1766.

Neuper C, Scherer R, Reiner M, Pfurtscheller G (2005) Imagery of motor actions: Differential effects of kinesthetic and visual-motor mode of imagery in singletrial EEG. Cognitive Brain Research 25:668-677.

Peck T, Seinfeld S, Aglioti S, Slater M (2013) Putting yourself in the skin of a black avatar reduces implicit racial bias. Consciousness and Cognition 22:779-787.

Perry A, Bentin S, Bartal I-A, Lamm C, Decety J (2010) "Feeling" the pain of those who are different from us: Modulation of EEG in the mu/alpha range. Cognitive, Affective, \& Behavioral Neuroscience 10:493-504.

Petkova VI, Ehrsson HH (2008) If I were you: perceptual illusion of body swapping. PLoS One 3:e3832.

Petkova VI, Khoshnevis M, Ehrsson HH (2011) The perspective matters! Multisensory integration in ego-centric reference frames determines full-body ownership. Frontiers in psychology 2.

Pfurtscheller G, Lopes da Silva FH (1999) Event-related EEG/MEG synchronization and desynchronization: basic principles. Clinical neurophysiology : official journal of the International Federation of Clinical Neurophysiology 110:18421857.

Preston SD, de Waal FBM (2002) Empathy: Its ultimate and proximate bases. Behavioral and Brain Sciences 25:1-20.

Sanchez-Vives MV, Slater M (2005) From presence to consciousness through virtual reality. Nature Reviews Neuroscience 6:332-339.

Sanchez-Vives MV, Spanlang B, Frisoli A, Bergamasco M, Slater M (2010) Virtual Hand Illusion Induced by Visuomotor Correlations. PLoS ONE 5:e10381.

Slater M, Pérez Marcos D, Ehrsson H, Sanchez-Vives MV (2008) Towards a digital body: the virtual arm illusion. Frontiers in Human Neuroscience 2.

Slater M, Spanlang B, Sanchez-Vives MV, Blanke O (2010) First Person Experience of Body Transfer in Virtual Reality. PLoS ONE 5:e10564.

Steptoe W, Steed A, Slater M (2013) Human tails: ownership and control of extended humanoid avatars. Ieee Transactions on Visualization and Computer Graphics 19:in press.

Swart H, Hewstone M, Christ O, Voci A (2010) The impact of crossgroup friendships in South Africa: Affective mediators and multigroup comparisons. Journal of Social Issues 66:309-333.

Tecchia F, Carrozzino M, Bacinelli S, Rossi F, Vercelli D, Marino G, Gasparello P, Bergamasco M (2010) A Flexible Framework for Wide-Spectrum VR Development. Presence: Teleoperators and Virtual Environments 19:302-312.

Tsakiris M, Haggard P (2005) The rubber hand illusion revisited: visuotactile integration and self-attribution. Journal of Experimental Psychology-Human Perception and Performance 31:80-91. 
Whitmarsh S, Nieuwenhuis ILC, Barendregt H, Jensen O (2011) Sensorimotor alpha activity is modulated in response to the observation of pain in others. Frontiers in Human Neuroscience 5.

Yang C-Y, Decety J, Lee S, Chen C, Cheng Y (2009) Gender differences in the mu rhythm during empathy for pain: An electroencephalographic study. Brain Research 1251:176-184.

Yetkin FZ, Mueller WM, Hammeke TA, Morris GLI, Haughton VM (1995) Functional Magnetic Resonance Imaging Mapping of the Sensorimotor Cortex with Tactile Stimulation. Neurosurgery 36:921-925.

\section{List of Figures}

Figure 1. Real: the participant wearing the HMD and EEG cap. Virtual: the IVR with the gender-matched collocated virtual avatar. And the two experimental conditions seen by the participant when looking towards his hand from the 1PP: HAND) virtual hand stabbed by the knife; TABLE) virtual table stabbed by the knife (control condition).

Figure 2. Box plots showing the responses to the questionnaire. The thick lines are the medians, and the boxes are the interquartile ranges (IQR). The whiskers follow the standard convention of extending to 1.5 times the IQR or the maximal/minimal data point.

Figure 3. Grand averaged stimulus locked ERPs for six representative front, central and parietal electrode locations. A significant increase in the amplitude of the P450 is observed in the HAND condition mainly at C3 and CP3 locations. Baseline from [$200 \mathrm{~ms}$ to $0 \mathrm{~ms}$ ], time 0 indicates the stimuli onset; a low pass filter $12 \mathrm{~Hz}$ halfamplitude cutoff was applied.

Figure 4. a) Time Frequency Evolution of the two conditions and the difference in the spectral activity. b) Grand averaged 1-s short time power spectra calculated 
from EEG data (electrode $\mathrm{C} 3$ ) recorded. The baseline corresponds to the range [-1 to 0] seconds before the stimuli and the activity period corresponds to the range [0.7 to 1.7] seconds after the stimuli. Both the Baseline and TABLE frequency spectra show a peak in the mu-rhythm that is attenuated in the HAND condition. c) Grand averaged Mu-rhythm (9-12Hz) Event Related Desynchronization for the C3 electrode. d) Grand averaged Readiness Potential (C3-C4) subtraction between the brain activity in the two hemispheres shows movement preparation effects. Low pass filter $8 \mathrm{~Hz}$, halfamplitude cutoff.

Figure 5. Path analysis for $\mathrm{P} 450$ and mu and in relation to questionnaire variables Harm Hand, Harm Table, Body Threat, Ownership and condition (Knife out $=0$, Knife in $=1$ ). The values on the paths are the path coefficients and the corresponding significance levels. The epsilon terms represent the random error term. The diagram can be interpreted as a set of simultaneous linear prediction equations, e.g. from Table 3 we can see that $\mathrm{P} 450=-1.50+1.69 *$ condition $+1.08 *($ Harm Hand $)-$ 0.46*(Harm Table $)+$ epsilon. The circles are the random error terms and values by the epsilon circles are their variances. The curved path represents a covariance. 


\section{List of Tables}

Table 1

Spearman Correlation Coefficients Between the Questionnaire Scores

\begin{tabular}{|l|c|c|c|c|c|}
\hline & Ownership & Harm Hand & Harm Table & No Ownership & Body Threat \\
\hline & & & & & \\
\hline Ownership & 1.000 & & & & \\
\hline & & & & & \\
\hline & 0.726 & 1.000 & & & \\
\hline & $(0.000)$ & & & & \\
\hline & & & & & \\
\hline & 0.162 & 0.302 & 1.000 & & \\
\hline & $(0.508)$ & $(0.209)$ & & & \\
\hline No Ownership & -0.048 & 0.079 & -0.125 & 1.000 & \\
\hline & $(0.844)$ & $(0.749)$ & $(0.611)$ & & \\
\hline & & & & & \\
\hline & & & & & \\
\hline & 0.481 & 0.774 & 0.418 & -0.179 & 1.000 \\
\hline & $(0.037)$ & $(0.000)$ & $(0.075)$ & $(0.463)$ & \\
\hline
\end{tabular}

Table 1 footnote: ( $\mathrm{P}$ values for test of 0 correlation). $\mathrm{P}=0.000$ means $\mathrm{P}<0.0005, \mathrm{n}=$ 19 
Table 2

Spearman Correlation Coefficients Between the Questionnaire Scores and EEG Variables

\begin{tabular}{|l|c|c|c|c|c|l|l|}
\hline & Ownership & $\begin{array}{l}\text { Harm } \\
\text { Hand }\end{array}$ & $\begin{array}{l}\text { Harm } \\
\text { Table }\end{array}$ & $\begin{array}{l}\text { No } \\
\text { Ownership }\end{array}$ & $\begin{array}{l}\text { Body } \\
\text { Threat }\end{array}$ & P450 & mu \\
\hline$p 450$ & 0.287 & 0.389 & 0.113 & -0.021 & 0.289 & 1.0000 & \\
\hline & $(0.0000)$ & $(0.0000)$ & $(0.089)$ & $(0.751)$ & $(0.0000)$ & & \\
\hline & & & & & & & \\
\hline$m u$ & 0.266 & 0.093 & -0.035 & -0.169 & 0.040 & 0.029 & 1.0000 \\
\hline & $(0.0000)$ & $(0.160)$ & $(0.601)$ & $(0.011)$ & $(0.545)$ & $(0.658)$ & \\
\hline
\end{tabular}

Table 2 footnote: $\mathrm{n}=228$. Overall $\mathrm{R}^{2}=0.26, \mathrm{~F}(5,222)=15.59, \mathrm{n}=228$.

Shapiro-Wilk test for normality of residuals: $\mathrm{P}=0.10$.

$$
\mathrm{P}=0.000 \text { means } \mathrm{P}<0.0005
$$


Table 3

Path Analysis for P450 and mu

\begin{tabular}{|c|c|c|c|c|c|}
\hline & Coef. & Std. Err. & $\mathbf{Z}$ & $\mathbf{P}$ & $\mathbf{r}, \mathbf{P}$ \\
\hline P450 & & & & & $\begin{array}{c}0.53 \\
\mathrm{P}=0.0000\end{array}$ \\
\hline condition & 1.69 & 0.35 & 4.84 & 0.000 & \\
\hline Harm Hand & 1.08 & 0.15 & 7.26 & 0.000 & \\
\hline Harm Table & -0.46 & 0.23 & -1.96 & 0.050 & \\
\hline Const. & -1.50 & 0.57 & -2.63 & 0.009 & \\
\hline mu & & & & & $\begin{array}{c}0.45 \\
\mathrm{P}=0.0000\end{array}$ \\
\hline condition & -0.14 & 0.02 & -7.23 & 0.000 & \\
\hline Harm Hand & 0.01 & 0.01 & 2.05 & 0.040 & \\
\hline Const. & -0.06 & 0.02 & -2.42 & 0.015 & \\
\hline \multicolumn{6}{|l|}{ Harm Hand } \\
\hline Ownership & 2.71 & 0.22 & 12.14 & 0.000 & \\
\hline \multicolumn{6}{|l|}{ Harm Table } \\
\hline Ownership & 0.66 & 0.22 & 3.02 & 0.003 & \\
\hline \multicolumn{6}{|l|}{ Body Threat } \\
\hline Ownership & 1.56 & 0.22 & 6.95 & 0.000 & \\
\hline
\end{tabular}

Table 3 footnote: Condition $=0$ (TABLE), 1 (HAND). r, P are the Pearson correlations and significance levels between fitted and observed values of the response variables. $\mathrm{P}=0.00 * 0$ means $\mathrm{P}<0.00 * 05$. 
Table 4

Regression for RP

\begin{tabular}{|l|c|c|c|l|}
\hline & Coef. & Std. Err. & $\mathrm{t}$ & $\mathrm{P}>\mathrm{t}$ \\
\hline & & & & \\
\hline condition & -0.87 & .38 & -2.28 & 0.029 \\
\hline Harm Table & 0.34 & .16 & 2.18 & 0.036 \\
\hline Const. & -0.10 & .41 & -0.25 & 0.802 \\
\hline
\end{tabular}

Table 4 footnote: Condition $=0$ (TABLE), $1($ HAND) $\mathrm{F}(2,35)=8.48, \mathrm{R} 2=0.17, \mathrm{P}=$ $0.001, \mathrm{n}=38$, Shapiro-Wilk (test for normality of residual errors) $\mathrm{P}=0.24$ 